\title{
La motivación. Un factor determinante en el aprendizaje
}

\author{
Sergio García-Jiménez ${ }^{1}$ \\ Instituto de Enseñanza Secundaria Jiménez de \\ la Espada, Cartagena (Murcia)
}

\begin{abstract}
Resumen: ¿Qué factores influyen en la motivación de nuestros alumnos? ¿Puede un alumno estar motivado para aprender si no tiene sus necesidades básicas cubiertas? ¿Es la motivación determinante en el proceso de aprendizaje de nuestro alumnado? En el presente documento trataremos estas y otras cuestiones relacionadas con la motivación. Veremos que la motivación puede venir de dos formas, extrínseca o intrínsecamente y trataremos las teorías de la motivación de Maslow. Finalmente hablaremos sobre los factores que determinan la motivación en el aula.

Palabras clave: Motivación intrínseca, Motivación extrínseca, Aprendizaje, Maslow, Motivación en el aula.

Abstract: What are the factors which influence the motivation of our students? Can students be motivated to learn if they do not have their basic needs covered? Is motivation a necessary factor in the learning process of our students? This document discusses these and other issues related to motivation. We will see that motivation can come in two ways, extrinsic or intrinsic and discuss theories of Maslow's motivation. Finally, we will talk about the factors which establish the motivation in the classroom.
\end{abstract}

Keywords: Intrinsic motivation, Extrinsic motivation, Learning, Maslow, Motivation in the classroom.

\section{Introducción}

La motivación es un concepto muy utilizado en el ámbito escolar, en el que suele emplearse para explicar la cantidad de esfuerzo que un alumno emplea en realizar diversas actividades (Entwistle, 1988). El hecho de que un alumno sea capaz de aprender una determinada materia por poseer unos conocimientos previos suficientes y unas aptitudes y estrategias adecuadas no basta para que el alumno inicie y lleve a cabo el proceso de aprendizaje. Para ello es necesario, además, poner en actividad ese proceso, y esto depende de la motivación, que puede definirse como la fuerza que impulsa y orienta la actividad de los individuos a conseguir un objetivo.

Una de las razones por la cual es importante el análisis de la motivación académica es su incidencia significativa en el aprendizaje escolar (Arias \& Rusillo, 2004).

\section{Motivación}

La motivación es un fenómeno complejo que está condicionado por innumerables factores (Palermo, 2005). Motivo, motor y motivación tienen la misma raíz que implica acción. La palabra motivación deriva del vocablo latín "movere", que significa movimiento, motivación significa moverse hacia. Se asocia la motivación con la forma en que la conducta se inicia, se energiza, se sostiene, se dirige y con el tipo de reacción subjetiva que está presente cuando realizamos una actividad. Podría decirse que está relacionada, haciendo analogías, con la energía de activación necesaria para vencer la inercia de no involucrarse en una tarea.

\footnotetext{
${ }^{1}$ Ingeniero en Organización Industrial. Profesor de enseñanza secundaria de la asignatura Tecnología en el IES Jiménez de la Espada de Cartagena (Murcia). Doctor por la Universidad de Alicante.
} 
Cualquier intento definitorio de motivación debería referirse a un proceso adaptativo, que es el resultado de un estado interno de un organismo, que le impulsa y le dirige hacia una acción en un sentido determinado. Es decir, existe una influencia de los factores externos y de los factores internos que activan al organismo, y le dirigen hacia la consecución de algún objetivo o meta que le es gratificante (Palermo, 2005).

Progresivamente, se ha ido aceptando que no se trata de una dimensión homogénea o monolítica, sino que adopta diferentes formas. Desempeña un papel fundamental en la ejecución de cualquier actividad. Para que el aprendizaje se produzca de una manera eficaz es necesaria la motivación, además, sin ella difícilmente puede haber aprendizaje. Es por ello importante conocer el papel que desempeña en el aprendizaje de los alumnos en el aula, a fin de ser capaces de diseñar actividades que la estimulen de forma adecuada.

Es necesario conocer y saber explicar cómo afectan las experiencias de aprendizaje a la motivación de los alumnos, extrayendo algunas implicaciones educativas que se deriven de las principales teorías de la motivación. Algunos autores exponen que, para aprender eficazmente son necesarias dos condiciones en el alumno: poder y querer (Ausubel, Novak, \& Hanesian, 1968). El poder es un problema de capacidad: de conocimientos previos, de inteligencia, de estilo cognitivo o intelectual. El querer es un problema de motivación: de los motivos que tenga el alumno, de sus expectativas, de sus actitudes e intereses. Las variables cognitivas afectan de una manera directa y específica en el aprendizaje, mientras que las variables motivacionales intervienen facilitando en el alumno el incremento y concentración de la atención y de la disposición, la persistencia ante las tareas y la tolerancia ante la frustración.

En el ámbito educativo, la falta de motivación suele mencionarse cuando algo falla en el proceso de aprendizaje (Heredia, 1999). Muchos profesores se quejan del poco aliciente que despierta en ellos la actividad docente que ejercitan a diario, atribuyendo su falta de interés al bajo nivel motivacional de los estudiantes.

En cualquier caso, la motivación académica es un complejo problema en el que saturan muchos factores y sus interacciones y que tiene un difícil abordaje psicopedagógico y educativo, y en el que es necesario abrir un amplio debate en la comunidad educativa (Cavero, 2006).

\subsection{Motivación intrínseca y extrínseca}

La motivación es la palanca que mueve toda conducta, lo que nos permite provocar cambios tanto a nivel escolar como de la vida en general (Bacete \& Betoret, 1997). Si nos trasladamos al contexto escolar y consideramos el carácter intencional de la conducta humana, parece bastante evidente que las actitudes, percepciones, expectativas y representaciones que tenga el estudiante de sí mismo, de la tarea a realizar, y de las metas que pretende alcanzar constituyen factores de primer orden que guían y dirigen la conducta del estudiante en el ámbito académico. Pero para realizar un estudio completo e integrador de la motivación, no sólo se debe tener en cuenta estas variables personales e internas sino también aquellas otras externas, procedentes del contexto en el que se desenvuelven los estudiantes, que les están influyendo y con los que interactúan.

Para Lamas Rojas (2008), parece haber coincidencia entre los distintos autores en vincular a la motivación intrínseca con aquellas acciones realizadas por el interés que genera la propia actividad, considerada como un fin en sí misma y no como un medio para alcanzar otras metas. En cambio, la orientación motivacional extrínseca, se caracteriza generalmente como aquella que lleva al individuo a realizar una 
determinada acción para satisfacer otros motivos que no están relacionados con la actividad en sí misma, sino más bien con la consecución de otras metas que en el campo escolar suelen fijarse en obtener buenas notas, lograr reconocimiento por parte de los demás, evitar el fracaso, ganar recompensas, etc.

La motivación de los alumnos para el aprendizaje, al igual que la motivación que impulsa a los individuos para cualquier actividad, puede ser intrínseca o extrínseca (Entwistle, 1988).

La motivación es intrínseca cuando los motivos que conducen al alumno hacia el aprendizaje son inherentes al propio alumno. Así, si un estudiante realiza una determinada tarea alentado por motivos internos, como pueden ser la satisfacción que le produce la propia actividad, o el conocimiento que consigue, o el hecho de descifrar un interrogante o un enigma, se dice que la motivación es intrínseca. $\mathrm{Si}$, por el contrario, el motivo que impulsa a aprender es ajeno al propio aprendizaje, esto es, si viene determinado por incentivos o reforzadores positivos o negativos externos al propio alumno, entonces se dice que la motivación es extrínseca. Cuando un alumno trabaja no por el placer que encuentra en la tarea, sino por la satisfacción de conseguir un aprobado o una calificación elevada, o por satisfacer las expectativas de sus padres o profesores, entonces su motivación es extrínseca. Mientras que, en ocasiones, los alumnos pueden mostrar interés por el contenido (motivación intrínseca), en otras (motivación extrínseca) buscan recompensas en forma de notas o calificaciones.

La distinción entre motivación intrínseca y extrínseca dista mucho de ser tan nítida como podría pensarse. Se ha visto que tanto la motivación intrínseca como la extrínseca descienden cuando el individuo recibe información negativa sobre sus capacidades y cuando percibe que no puede mejorar. Por otro lado, es obvio que la dedicación a un tema interesante para el sujeto puede incrementarse con refuerzos externos o extinguirse en ausencia de éstos (Ayala, Martínez, \& Yuste, 2004).

Algunos autores exponen que, dentro de la psicología científica han aparecido distintas explicaciones de la motivación (Rodriguez \& Luca de Tena, 2001): las explicaciones conductistas dicen que la conducta es impulsada por necesidades y por reforzadores externos, las explicaciones psicoanalíticas defienden que el comportamiento está impulsado por fuerzas instintivas inconscientes, las explicaciones cognitivas vienen a destacar la importancia de las percepciones y de las expectativas del individuo en la conducta, y las explicaciones humanísticas se caracterizan por concebir al hombre con unas necesidades jerarquizadas que le impulsan a la búsqueda y desarrollo de su propia identidad, a su autorrealización personal. Dentro de estas explicaciones de la motivación, se encuentran algunas teorías específicas que han conseguido gran relieve e influencia, como la Teoría de Maslow, la Teoría de la motivación de logro y la Teoría Atribucional de Weiner.

\subsection{Teoría de la motivación de Maslow}

La teoría de Maslow (1968) se engloba dentro de las explicaciones humanísticas, y se basa en la idea de que la satisfacción de las necesidades es el principio elemental que subyace en el desarrollo de la persona.

Maslow señala siete necesidades básicas, ordenadas jerárquicamente, con un doble sentido (Maslow, Frager, Fadiman, McReynolds, \& Cox, 1970): por un lado, nos dice que las necesidades de cada nivel sólo aparecen si han sido satisfechas las de los niveles anteriores; y, por otro, que el proceso evolutivo de aparición y desarrollo de las necesidades en los seres humanos se produce siguiendo el siguiente orden: necesidades fisiológicas, de seguridad, de pertenencia y afecto, de autoestima, de logro intelectual, de apreciación estética y de autorrealización. 


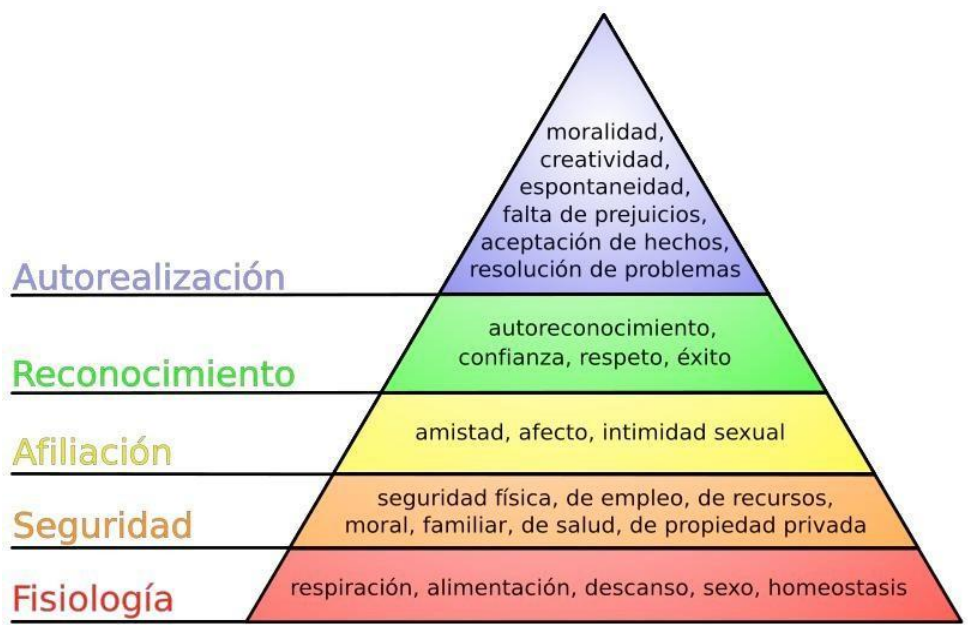

Figura 1. Pirámide de Maslow.

1. Necesidades fisiológicas: necesidad de alimento, beber, dormir, etc. Estas necesidades son las más imperiosas y, si no son satisfechas, dominan toda la conducta del individuo y no dejan lugar a ningún otro tipo de motivaciones.

2. Necesidad de seguridad física y psicológica: necesidad de apoyo y de contar con un ambiente ordenado y justo, ausente de peligros físicos y psicológicos. Este grupo de necesidades, junto con las del primer nivel, cuando no son suficientemente satisfechas, controlan la conducta por completo e impiden que aparezcan las necesidades que están por encima de ellas.

3. Necesidad de pertenencia y afecto: necesidad de integración y de afecto dentro de un grupo social, como la familia o el grupo de compañeros. Cuando las necesidades fisiológicas y de seguridad están relativamente satisfechas, surgen estas necesidades sociales de pertenencia y de afecto.

4. Necesidad de autoestima: necesidad de tener una imagen positiva de sî mismo basada en la aprobación y el reconocimiento por parte de los demás. Su satisfacción provoca sentimientos de confianza en sí mismo, de dignidad, de fuerza, de competencia y de capacidad. Por el contrario, si estas necesidades no encuentran satisfacción, surgen sentimientos de inferioridad y de fracaso, lo que provoca la inactividad y el abandono.

5. Necesidad de logro intelectual: necesidad de curiosidad, de conocer y comprender el mundo. En un peldaño superior aparece en el individuo la necesidad de conocer, de conseguir explicaciones y de entender, y se siente atraído por lo misterioso y por lo desconocido.

6. Necesidad de apreciación estética: búsqueda del orden y de la belleza. En muchos individuos está presente una necesidad estética que se pone de manifiesto en un impulso hacia el orden, la simetría, en la terminación de las cosas emprendidas y en la búsqueda de la belleza. 
7. Necesidad de autorrealización: El individuo siente la necesidad de hacer aquello para lo que se siente dotado y de desarrollar sus propias posibilidades y se siente contento y satisfecho cuando tiene la oportunidad de desplegar esas posibilidades dentro de su vida familiar, escolar o profesional.

Maslow (1968) agrupa estas siete necesidades en dos categorías: necesidades de deficiencia, en la que se incluyen las de los cuatro primeros niveles, y necesidades de ser, que recoge las tres necesidades de los tres niveles superiores. Las primeras se caracterizan porque, cuando no son atendidas, se incrementa la motivación para hallar la forma de satisfacerlas y dominan por completo la conducta del individuo y no dejan lugar a que aparezca ningún otro tipo de necesidades. Todas las necesidades de deficiencia, cuando son satisfechas, se extinguen y dejan paso a que aparezcan las necesidades de logro intelectual, de apreciación estética y de autorrealización. Estas necesidades de ser no se extinguen cuando son atendidas, sino que aumentan: cuanto mayores son los logros de una persona dentro de un área de conocimientos, mayor es el incremento de su necesidad por seguir ampliando su saber y su comprensión en ese o en otro campo de conocimientos.

Este planteamiento tiene importantes implicaciones educativas, pues ofrece un marco de referencia para reflexionar sobre la motivación de los alumnos. Como expone Woolfolk (1987), es cierto que los esfuerzos de los alumnos por satisfacer las necesidades de los niveles inferiores pueden en ocasiones inhibir sus impulsos de lograr objetivos de los niveles superiores. No es de esperar que los estudiantes que no tienen cubiertas sus necesidades básicas, como el alimento, la salud y el afecto, que se sientan motivados para el aprendizaje y la adquisición de conocimientos. Tampoco es de esperar que aparezca una motivación fuerte para el estudio en aquellos alumnos que se sienten temerosos, inseguros, escasamente aceptados o integrados en su grupo o con baja autoestima y sin confianza en sí mismos.

Una de las consecuencias prácticas más inmediatas es que el profesor debe hacer todo lo posible para procurar que las necesidades de nivel inferior de sus alumnos estén cubiertas, de manera que tengan mayores posibilidades de desarrollarse las de niveles superiores. Por ejemplo, es más probable que los estudiantes busquen la satisfacción de la necesidad de comprender y conocer si están físicamente cómodos, se sienten seguros y relajados, si tienen una sensación de pertenencia al grupo y experimentan un sentido de autoestima positivo.

Evidentemente, aunque el profesor haga todo lo que pueda para satisfacer las necesidades de nivel inferior de esta jerarquía, es muy difícil que pueda controlar determinados aspectos, por ejemplo, es poco probable que los estudiantes que van a la escuela hambrientos o enfermos estén motivados para buscar conocimiento; del mismo modo, un alumno puede considerar que sus padres no lo quieren o que sus compañeros no lo aceptan, al tener sus necesidades de amor y pertenencia no satisfechas, es menos probable que esté motivado para aprender.

La jerarquía de Maslow también ayuda a reflexionar sobre la conducta de los estudiantes. Sus deseos para cubrir las necesidades del nivel inferior pueden a veces entrar en conflicto con el deseo del profesor de que alcancen metas del nivel superior. Por ejemplo, pertenecer a un grupo social y mantener su estima dentro de éste, es importante para los estudiantes, especialmente durante la adolescencia. Si por hacer lo que el profesor dice se genera un conflicto con las reglas del grupo, los estudiantes pueden optar por ignorar los deseos del profesor e incluso desafiarlos. 
La principal crítica que ha recibido esta teoría ha sido por la razón obvia de que la gente no siempre parece comportarse como predice. La mayoría de nosotros tenemos diferentes tipos de necesidades y podemos estar motivados por muchas de ellas al mismo tiempo, podemos incluso anteponer una necesidad superior a una inferior, por ejemplo, no dormir o cenar porque estamos estudiando. A pesar de que puede haber excepciones a este desarrollo jerárquico y que en determinados casos pueden darse conflictos entre algunos niveles, podemos considerar que el esquema de Maslow es en líneas generales válido.

\section{La motivación en el aula}

Los profesores se encuentran en su labor docente con un gran problema: la falta de motivación de muchos de sus alumnos quienes, con frecuencia, están más preocupados por aprobar que por aprender (Álvarez, Mieres, \& Rodríguez, 2008). Ante esta situación, los profesores deben plantearse qué pueden hacer para mejorar la motivación y el interés de sus alumnos por el aprendizaje.

Como exponen Rodríguez y Luca de Tena (2001), la motivación es un tema difícil de analizar porque son muchos los factores que favorecen o interfieren en el deseo por aprender: la naturaleza de la tarea de aprendizaje, las características de cada alumno, el ambiente de la clase, la personalidad del profesor, el estilo educativo de la familia, etc. Todos ellos son los factores que determinan si el alumno estará o no motivado para aprender:

1. Factores relacionados con las características del alumno. Toda conducta de aprendizaje está basada en diferentes características personales del sujeto que inciden en su mayor o menor motivación intrínseca. Estas características, que son determinantes tanto en la conducta del sujeto como en su forma de evaluar a posteriori su actuación, podemos clasificarlas en tres niveles: el afectivo, donde se encuentran los sentimientos y emociones positivos o negativos respecto a la escuela, las tareas escolares y el aprendizaje en general (actitudes), y la mayor o menor autoestima; el cognitivo, donde se manifiestan las expectativas de éxito o de fracaso (atribuciones) y las características propias del sujeto como la curiosidad o el interés; y el de personalidad, con la modificación del autoconcepto y la satisfacción o no de las necesidades del yo.

2. Factores relacionados con el tipo de aprendizaje. Las personas no funcionamos igual ante cualquier tipo de aprendizaje, sino que existen situaciones más o menos eficaces dependiendo de factores como el nivel de estimulación que proporcionan, la complejidad y dificultad que presentan, el hecho de que se trate de contenidos intrínsecamente significativos, de la forma en cómo nos son presentados, o del tipo de evaluación que esperemos tener después del aprendizaje.

3. Factores relacionados con los compañeros. El grupo de iguales ofrece posibilidades de interacción que permitirán desarrollar capacidades como la empatía, ser capaz de reconocer los sentimientos ajenos y establecer relaciones afectivas y emocionales, pero además ofrece información al sujeto sobre sí mismo. Los alumnos valoran su nivel de competencia teniendo en 
cuenta sus resultados personales al compararse con el rendimiento de sus compañeros, lo que les ayudará a desarrollar, mantener y/o modificar su autoconcepto, repercutiendo sobre su motivación y su comportamiento académico.

El sistema de organización social del aula utilizado preferentemente por el profesor (organización competitiva, cooperativa o individualista), determinará diferentes modos de interacción entre los alumnos que influirán en la implicación en la tarea, el compromiso, el esfuerzo mantenido, la satisfacción personal lograda y, en definitiva, en la motivación.

4. Factores relacionados con el profesor. El profesor continúa siendo una pieza clave en el proceso de enseñanza-aprendizaje, con su actuación puede facilitar la aparición de la motivación académica o, por el contrario, llevar a los alumnos al desánimo.

Tanto los aspectos de índole personal (la empatía, la amabilidad en el trato, el sentido de su eficacia, etc.), como los didácticos (organización del material, claridad en las exposiciones, etc.) y los de interrelación (tipo de disciplina, aplicación de recompensas, etc.) influyen en la aparición y posterior mantenimiento del interés hacia la materia que imparte y hacia el aprendizaje en general.

5. Factores relacionados con la familia. Todos somos conscientes de la importancia que las actitudes, expectativas y estilo de crianza de los padres tienen en el tipo y nivel de motivación escolar de sus hijos. A medida que el niño crece los padres colaboran en el desarrollo de su autoconcepto académico a través de su nivel de implicación que hace referencia a comentarios sobre su capacidad, al aliento que le infunden ante sus proyectos, al apoyo que le ofrecen, a la valoración de la educación y al tipo de relación que mantienen con la escuela.

Por otra parte, el estilo educativo de los padres, la forma de educar a sus hijos, influirá directamente en el desarrollo de su personalidad afectando a aspectos como la autorregulación, independencia, confianza en sí mismo, etc. que determinarán su motivación escolar.

Para Alonso Tapia (2005), los alumnos no sólo buscan incrementar su saber y sus capacidades y experimentar su competencia, sino también poder ayudar a otros y sentir el apoyo del profesor, metas cuyo efecto se multiplica en la medida en que el alumno está dispuesto a esforzarse, pero todo ello siempre y cuando se perciba la utilidad de lo que se ha de aprender.

El docente debe conocer las líneas generales de todas estas influencias ya que en muchos casos le servirán como orientación para poder determinar los factores implicados en la motivación de sus alumnos y poder planificar unas formas de actuación conjunta con los padres. En este sentido cada día preocupa más a la institución escolar contar con el apoyo y la colaboración de la familia con la que deberían compartirse los criterios educativos. 


\section{Referencias bibliográficas}

Álvarez, B. Á, Mieres, C. G., \& Rodríguez, N. G. (2008). La motivación y los métodos de evaluación como variables fundamentales para estimular el aprendizaje autónomo. Revista De Docencia Universitaria, 1(2)

Arias, P. F. C., \& Rusillo, M. T. C. (2004). Diferencias de género en la motivación académica de los alumnos de educación secundaria obligatoria. Electronic Journal of Research in Educational Psychology, 2(3), 97-112.

Ausubel, D. P., Novak, J. D., \& Hanesian, H. (1968). Educational psychology: A cognitive view.

Ayala, C. L., Martínez, R., \& Yuste, C. (2004). Cuestionario de estrategias de aprendizaje yMotivación(CEAM). Madrid: Instituto de Orientación Psicológica EOS.

Bacete, F. J. G., \& Betoret, F. D. (1997). Motivación, aprendizaje rendimiento escolar. Reme, 1,3 .

Cavero, M. Á B. (2006). Motivación y rendimiento académico en alumnos de educación secundaria obligatoria y bachillerato LOGSE. Revista De Educación, (340), 379-414.

Entwistle, N. (1988). La comprensión del aprendizaje en el aula Barcelona: Paidós/MEC.

Heredia, E. B. (1999). Marco conceptual e investigación de la motivación humana. Reme, 2(1), 4.

Lamas Rojas, H. (2008). Aprendizaje autorregulado, motivación y rendimiento académico. Liberabit, 14(14), 15-20.

Maslow, A. H. (1968). Toward a psychology of being. Princeton, New York: Van Nostrand.

Maslow, A. H., Frager, R., Fadiman, J., McReynolds, C., \& Cox, R. (1970). Motivation and personality Harper \& Row New York.

Palermo, M. M. (2005). El núcleo de una estrategia didáctica universitaria: Motivación y comprensión. Revista ieRed: Revista Electrónica De La Red De Investigación Educativa, 1(3), 1.

Rodriguez, R. I., \& Luca de Tena, C. (2001). Programa de motivación la enseñanza secundaria obligatoria. ¿Cómo puedo mejorar la motivación de mis alumnos? Málaga: Ed. Aljibe.

Tapia, J. A. (2005). Motivaciones, expectativas y valores-intereses relacionados con el aprendizaje: El cuestionario MEVA. Psicothema, 17(3), 404-411.

Woolfolk, A. E. (1987). Educational Psychology. New Jersey: Prentice-Hall. 